\title{
Study of the XYZ states at the BESIII
}

\author{
Chang-Zheng Yuan \\ Institute of High Energy Physics, Chinese Academy of Sciences, Beijing 100049, China \\ E-mail: yuancz@ihep.ac.cn \\ Received August 9, 2015; accepted August 27, 2015
}

\begin{abstract}
With its unique data samples at energies of 3.8-4.6 GeV, the BESIII experiment made a significant contribution to the study of charmonium and charmonium-like states, i.e., the XYZ states. We review the results for observations of the $Z_{c}(3900)$ and $Z_{c}(4020)$ states, the $X(3872)$ in $e^{+} e^{-}$annihilation, and charmonium $\psi\left(1^{3} D_{2}\right)$ state, as well as measurements of the cross-sections of $\omega \chi_{c J}$ and $\eta J / \psi$, and the search for $e^{+} e^{-} \rightarrow \gamma \chi_{c J}$ and $\gamma Y(4140)$. We also present data from BESIII that may further strengthen the study of the XYZ and conventional charmonium states, and discuss perspectives on future experiments.
\end{abstract}

Keywords charmonium, exotic states, $e^{+} e^{-}$annihilation, hadronic transitions, radiative transtitions

PACS numbers 14.40.Rt, 14.40.Pq, 13.25.Gv, 13.20.Gd, 13.66.Bc

\section{Contents}

1 Introduction

2 Charged charmonium-like states: $Z_{c} \mathrm{~s}$

2.1 Observation of $Z_{c}(3900)$ and $Z_{c}(3885)$

2.1.1 Observation of $Z_{c}(3900)$

2.1.2 Observation of $Z_{c}(3885)$

2.2 Observation of $Z_{c}(4020)$ and $Z_{c}(4025)$

2.2.1 Observation of $Z_{c}(4020)$

2.2.2 Observation of $Z_{c}(4025)$

3 Observation of $Y(4260) \rightarrow \gamma X(3872)$

4 Observation of $\psi\left(1^{3} D_{2}\right)$

5 Search for $e^{+} e^{-} \rightarrow \gamma \chi_{c J}$ and $e^{+} e^{-} \rightarrow \gamma Y(4140)$

6 Structures in $e^{+} e^{-} \rightarrow$ charmonium + hadrons

6.1 Observation of $e^{+} e^{-} \rightarrow \omega \chi_{c 0}$

6.2 Measurement of $e^{+} e^{-} \rightarrow \eta J / \psi$

7 More data for $X Y Z$ study

$8 X Y Z$ study in future experiments

9 Conclusion

Acknowledgements

References and notes

\section{Introduction}

Many charmonium and charmonium-like states were discovered at $B$-factories in the first decade of the 21 st century [1]. Whereas some of these are good charmonium candidates, as predicted in different models, many states have exotic properties, which may indicate that exotic states, such as multi-quark, molecule, hybrid, or hadronquarkonium, have been observed [2].

The BESIII experiment [3] at the BEPCII storage ring started its first collisions in the tau-charm energy region in 2008. After a few years running at suitable energies for its well-defined physics programs [4], i.e., at $J / \psi$ and $\psi(2 S)$ peaks in 2009 and the $\psi(3770)$ peak in 2010 and 2011, the BESIII experiment started to collect data for the study of the XYZ particles, which were not described in the Yellow Book [4].

As the design center-of-mass (c.m.) energy of the BEPCII was $2.0-4.2 \mathrm{GeV}$, there were not many options for data samples relevant to the XYZ-related physics. BESIII took its first data sample at the peak of $\psi(4040)$ in May 2011, with the aim of searching for the wellknown $X(3872)$ in the $\psi(4040)$ radiative transition and possibly the excited $P$-wave charmonium spin-triplet states in similar transitions. This sample is about 0.5 $\mathrm{fb}^{-1}$, which is limited by the one-month running time left after the $\psi(3770)$ data taken in the 2010-2011 run. Data were not collected at the $\psi(4170)$ peak because the CLEO-c experiment had already collected a sample of about $0.6 \mathrm{fb}^{-1}$ for the study of $D_{s}$ decays, which could be used for similar studies.

The upgrade of BEPCII's LINAC in summer 2012 in-

\footnotetext{
*Special Topic: Potential Physics at a Super $\tau$-charm Factory (Ed. Hai-Bo Li). Talk at the "International Workshop on Physics at Future High Intensity Collider 2-7 GeV in China", January 13-16, 2015, University of Science and Technology of China (USTC), Hefei, China.
} 
creased the highest beam energy from 2.1 to $2.3 \mathrm{GeV}$, making it possible to collect data at higher c.m. energies (up to $4.6 \mathrm{GeV}$ ). This made data collection possible at almost all known vector states, including $Y(4260)$, $Y(4360), \psi(4415)$, and (marginally) $Y(4660)$.

The data collected at a c.m. energy of $\sqrt{s}=4.26 \mathrm{GeV}$ turned out to be very fruitful. One month's data of 525 $\mathrm{pb}^{-1}$ (from December 14, 2012 to January 14, 2013) produced observations of the charged charmonium-like state $Z_{c}(3900)$ [5], resulting in changes to the data collection plan for the 2012-2013 run. More data were accumulated at c.m. energies of $4.26 \mathrm{GeV}$ and then $4.23 \mathrm{GeV}$. Data from the $Y(4360)$ peak were also obtained in spring 2013, and data from even higher energies (4.42 and 4.6 $\mathrm{GeV}$ ) were recorded in 2014 after a fine scan of the total hadronic cross-sections between 3.8 and $4.6 \mathrm{GeV}$ at more than 100 energy points, with a total integrated luminosity of about $800 \mathrm{pb}^{-1}$.

The dedicated data samples for the XYZ study are presented in Table 1, which lists the nominal c.m. energy, measured c.m. energy, and integrated luminosity at each energy point. These data were used for all the analyses presented in this article.

Table 1 The measured c.m. energy [6], integrated luminosity [7] of each data sample collected for the study of the XYZ states. The uncertainties on the integrated luminosities are statistical only; a $1 \%$ systematic uncertainty common to all the data points is not listed.

\begin{tabular}{ccc}
\hline Data sample & c.m. energy $(\mathrm{MeV})$ & $\mathcal{L}\left(\mathrm{pb}^{-1}\right)$ \\
\hline 3810 & $3807.65 \pm 0.10 \pm 0.58$ & $50.54 \pm 0.03$ \\
3900 & $3896.24 \pm 0.11 \pm 0.72$ & $52.61 \pm 0.03$ \\
4009 & $4007.62 \pm 0.05 \pm 0.66$ & $481.96 \pm 0.01$ \\
4090 & $4085.45 \pm 0.14 \pm 0.66$ & $52.63 \pm 0.03$ \\
4190 & $4188.59 \pm 0.15 \pm 0.68$ & $43.09 \pm 0.03$ \\
4210 & $4207.73 \pm 0.14 \pm 0.61$ & $54.55 \pm 0.03$ \\
4220 & $4217.13 \pm 0.14 \pm 0.67$ & $54.13 \pm 0.03$ \\
4230 & $4226.26 \pm 0.04 \pm 0.65$ & $1091.74 \pm 0.15$ \\
4245 & $4241.66 \pm 0.12 \pm 0.73$ & $55.59 \pm 0.04$ \\
4260 & $4257.97 \pm 0.04 \pm 0.66$ & $825.67 \pm 0.13$ \\
4310 & $4307.89 \pm 0.17 \pm 0.63$ & $44.90 \pm 0.03$ \\
4360 & $4358.26 \pm 0.05 \pm 0.62$ & $539.84 \pm 0.10$ \\
4390 & $4387.40 \pm 0.17 \pm 0.65$ & $55.18 \pm 0.04$ \\
4420 & $4415.58 \pm 0.04 \pm 0.72$ & $1073.56 \pm 0.14$ \\
4470 & $4467.06 \pm 0.11 \pm 0.73$ & $109.94 \pm 0.04$ \\
4530 & $4527.14 \pm 0.11 \pm 0.72$ & $109.98 \pm 0.04$ \\
4575 & $4574.50 \pm 0.18 \pm 0.70$ & $47.67 \pm 0.03$ \\
4600 & $4599.53 \pm 0.07 \pm 0.74$ & $566.93 \pm 0.11$ \\
\hline
\end{tabular}

\section{Charged charmonium-like states: $Z_{c} \mathrm{~s}$}

The BESIII experiment observed, for the first time, a charged charmonium-like state close to the $D \bar{D}^{*}$ thresh- old $Z_{c}(3900) / Z_{c}(3885)$, and a charged charmonium-like state close to the $D^{*} \bar{D}^{*}$ threshold $Z_{c}(4020) / Z_{c}(4025)$. Their neutral partners were also observed, confirming their isospins to be one.

\subsection{Observation of $Z_{c}(3900)$ and $Z_{c}(3885)$}

\subsubsection{Observation of $Z_{c}(3900)$}

The BESIII experiment studied the $e^{+} e^{-} \rightarrow \pi^{+} \pi^{-} J / \psi$ process at a c.m. energy of $4.26 \mathrm{GeV}$ using a $525 \mathrm{pb}^{-1}$ data sample [5]. A structure at around $3.9 \mathrm{GeV} / c^{2}$ was observed in the $\pi^{ \pm} J / \psi$ mass spectrum with a statistical significance larger than $8 \sigma$, which is referred to as the $Z_{c}(3900)$. A fit to the $\pi^{ \pm} J / \psi$ invariant mass spectrum (see Fig. 1), neglecting interference, results in a mass of $(3899.0 \pm 3.6 \pm 4.9) \mathrm{MeV} / c^{2}$ and a width of $(46 \pm 10 \pm 20) \mathrm{MeV}$. The associated production ratio was measured to be $R=\frac{\sigma\left(e^{+} e^{-} \rightarrow \pi^{ \pm} Z_{c}(3900)^{\mp} \rightarrow \pi^{+} \pi^{-} J / \psi\right)}{\sigma\left(e^{+} e^{-} \rightarrow \pi^{+} \pi^{-} J / \psi\right)}=$ $(21.5 \pm 3.3 \pm 7.5) \%$.

In the Belle experiments, the cross-section of $e^{+} e^{-} \rightarrow$ $\pi^{+} \pi^{-} J / \psi$ was measured from $3.8-5.5 \mathrm{GeV}$ using the initial state radiation (ISR) method. The intermediate states in $Y(4260) \rightarrow \pi^{+} \pi^{-} J / \psi$ decays were also investigated [8]. The $Z_{c}(3900)$ state (referred to as $Z(3900)^{+}$ in the Belle paper) with a mass of $(3894.5 \pm 6.6 \pm$ 4.5) $\mathrm{MeV} / \mathrm{c}^{2}$ and a width of $(63 \pm 24 \pm 26) \mathrm{MeV}$ was observed in the $\pi^{ \pm} J / \psi$ mass spectrum (see Fig. 1) with a statistical significance larger than $5.2 \sigma$.

The $Z_{c}(3900)$ state was confirmed shortly after with CLEO-c data at a c.m. energy of $4.17 \mathrm{GeV}$ [9], and the mass and width agreed very well with the BESIII and Belle measurements.

A neutral state $Z_{c}(3900)^{0} \rightarrow \pi^{0} J / \psi$ with a significance of $10.4 \sigma$ was observed at BESIII in $e^{+} e^{-} \rightarrow \pi^{0} \pi^{0} \mathrm{~J} / \psi$ with c.m. energy ranges from 4.19-4.42 GeV [10]. The mass and width were measured to be $(3894.8 \pm 2.3 \pm$ $3.2) \mathrm{MeV} / c^{2}$ and $(29.6 \pm 8.2 \pm 8.2) \mathrm{MeV}$, respectively. This state is interpreted as the neutral partner of the $Z_{c}(3900)^{ \pm}$, as it decays to $\pi^{0} J / \psi$ and its mass is close to that of $Z_{c}(3900)^{ \pm}$. This is in agreement with the previously reported $3.5 \sigma$ evidence for $Z_{c}(3900)^{0}$ in the CLEOc data [9]. The measured Born cross-sections of $e^{+} e^{-} \rightarrow$ $\pi^{0} \pi^{0} J / \psi$ were about half of those for $e^{+} e^{-} \rightarrow \pi^{+} \pi^{-} J / \psi$ measured in the Belle experiment [8], which is consistent with the isospin symmetry expectation.

\subsubsection{Observation of $Z_{c}(3885)$}

The $Z_{c}(3900)$ state observed in the $\pi J / \psi$ final state is close to and above the $D \bar{D}^{*}$ mass threshold. With the same data sample at $\sqrt{s}=4.26 \mathrm{GeV}$, the BESIII 

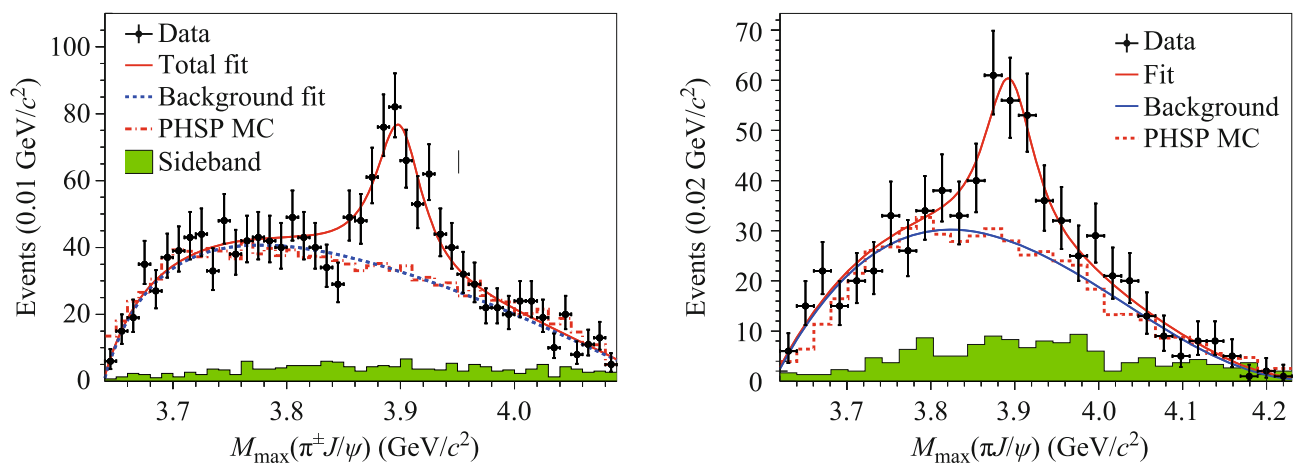

Fig. 1 Unbinned maximum likelihood fit to the distribution of the $M_{\max }(\pi J / \psi)$ (left panel from BESIII and right panel from Belle). Points with error bars are data, the curves are the best fit, the dashed histograms are the phase space distributions and the shaded histograms are the non- $\pi^{+} \pi^{-} J / \psi$ background estimated from the normalized $J / \psi$ sidebands.
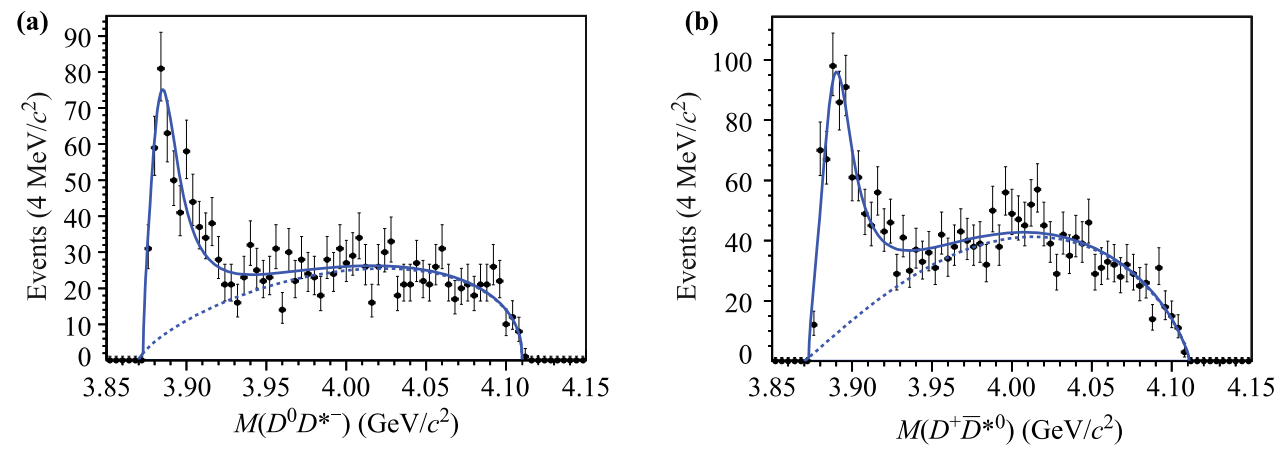

Fig. 2 The $M\left(D^{0} D^{*-}\right)$ (a) and $M\left(D^{+} \bar{D}^{* 0}\right)$ (b) distributions for selected events at $\sqrt{s}=4.26 \mathrm{GeV}$. The curves show the best fits.

experiment studied $e^{+} e^{-} \rightarrow \pi^{ \pm}\left(D \bar{D}^{*}\right)^{\mp}$. A structure (referred to as $\left.Z_{c}(3885)\right)$ was observed in the $\left(D \bar{D}^{*}\right)^{ \pm}$invariant mass distribution [11]. When fitted to a massdependent-width Breit-Wigner (BW) function, the pole mass and width were determined to be $(3883.9 \pm 1.5 \pm 4.2)$ $\mathrm{MeV} / \mathrm{c}^{2}$ and $(24.8 \pm 3.3 \pm 11.0) \mathrm{MeV}$, respectively (see Fig. 2). The angular distribution of the $Z_{c}(3885)$ system favors a $J^{P}=1^{+}$assignment for the structure and disfavors $1^{-}$or $0^{-}$. The production rate was measured to be $\sigma\left(e^{+} e^{-} \rightarrow \pi^{\mp} Z_{c}(3885)^{ \pm}\right) \times \mathcal{B}\left(Z_{c}(3885)^{ \pm} \rightarrow\left(D \bar{D}^{*}\right)^{ \pm}\right)=$ $(83.5 \pm 6.6 \pm 22.0) \mathrm{pb}$.

An important question is whether $Z_{c}(3885)$ is the same as $Z_{c}(3900)[5,8]$. The mass and width of $Z_{c}(3885)$ are $2 \sigma$ and $1 \sigma$, respectively, below those of $Z_{c}(3900)$, as observed by the BESIII and Belle experiments. However, neither fit considers the possibility of interference with a coherent non-resonant background, which could shift the results. A spin-parity quantum number determination for $Z_{c}(3900)$ would provide an additional test of this possibility.

Assuming the $Z_{c}(3885)$ structure is caused by $Z_{c}(3900)$, we obtain $\frac{\Gamma\left(Z_{c}(3885) \rightarrow D \bar{D}^{*}\right)}{\Gamma\left(Z_{c}(3900) \rightarrow \pi J / \psi\right)}=6.2 \pm 1.1 \pm 2.7$. This ratio is much smaller than typical values for decays of conventional charmonium states above the open charm threshold, e.g., $\Gamma(\psi(3770) \rightarrow D \bar{D}) / \Gamma(\psi(3770) \rightarrow$ $\left.\pi^{+} \pi^{-} J / \psi\right)=482 \pm 84 \quad[12]$ and $\Gamma(\psi(4040) \rightarrow$ $\left.D^{(*)} \bar{D}^{(*)}\right) / \Gamma(\psi(4040) \rightarrow \eta J / \psi)=192 \pm 27$ [13]. This suggests very different dynamics in the $Y(4260)$ $Z_{c}(3900)$ system.

\subsection{Observation of $Z_{c}(4020)$ and $Z_{c}(4025)$}

\subsubsection{Observation of $Z_{c}(4020)$}

BESIII measured $e^{+} e^{-} \rightarrow \pi^{+} \pi^{-} h_{c}$ cross-sections [14] at c.m. energies of $3.90-4.42 \mathrm{GeV}$. Intermediate states were studied by examining the Dalitz plot of the selected $\pi^{+} \pi^{-} h_{c}$ candidate events. The $h_{c}$ signal was selected using $3.518<M_{\gamma \eta_{c}}<3.538 \mathrm{GeV} / c^{2}$, and $\pi^{+} \pi^{-} h_{c}$ samples of 859 events at $4.23 \mathrm{GeV}, 586$ events at $4.26 \mathrm{GeV}$, and 469 events at $4.36 \mathrm{GeV}$ were obtained with purities of $65 \%$. Although there are no clear structures in the $\pi^{+} \pi^{-}$system, there is clear evidence for an exotic charmonium-like structure in the $\pi^{ \pm} h_{c}$ system, as clearly shown in the Dalitz plot. Figure 3(a) shows the projection of the $M\left(\pi^{ \pm} h_{c}\right)$ (two entries per event) distribution for the signal events, as well as the background events estimated from normalized $h_{c}$ mass sidebands. There is a significant peak at around $4.02 \mathrm{GeV} / c^{2}\left(Z_{c}(4020)\right)$, and there are also some events at around $3.9 \mathrm{GeV} / c^{2}$ (inset 

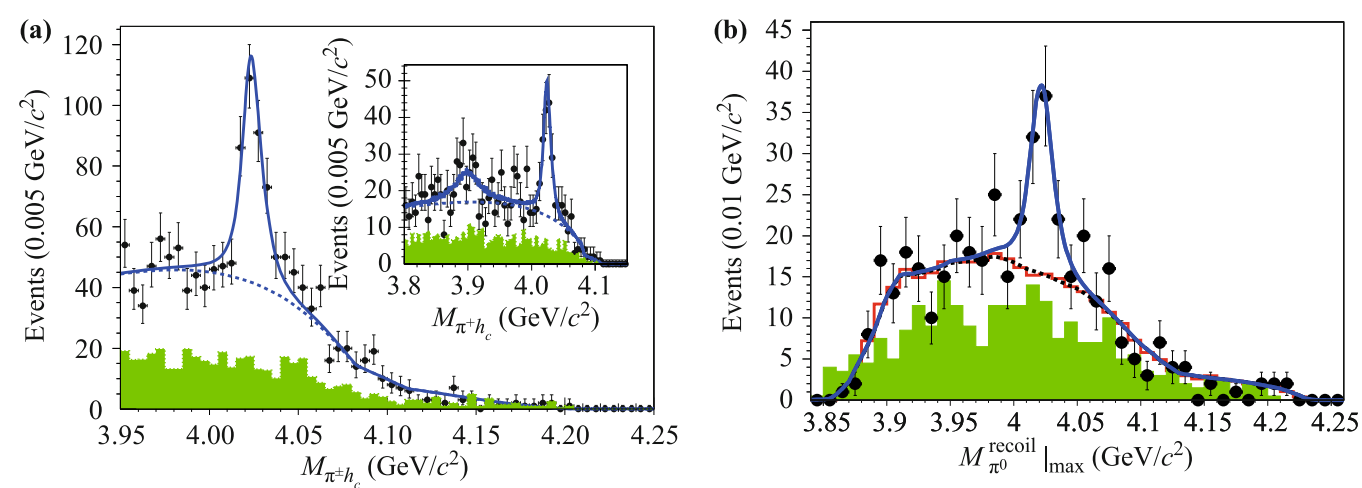

Fig. 3 Sum of the simultaneous fits to the $M\left(\pi^{ \pm} h_{c}\right)$ (a) and $M\left(\pi^{0} h_{c}\right)$ (b) distributions at $4.23,4.26$, and $4.36 \mathrm{GeV}$ in the BESIII data; the inset in the left panel shows the sum of the simultaneous fit to the $M_{\pi+h_{c}}$ distributions at 4.23 and 4.26 $\mathrm{GeV}$ with $Z_{c}(3900)$ and $Z_{c}(4020)$. Dots with error bars are data; shaded histograms are normalized sideband background; the solid curves show the total fit, and the dotted curves the backgrounds from the fit.

of Fig. 3(a)), which could be $Z_{c}(3900)$. The individual datasets at $\sqrt{s}=4.23,4.26$, and $4.36 \mathrm{GeV}$ show similar structures.

An unbinned maximum likelihood fit was applied to the $M\left(\pi^{ \pm} h_{c}\right)$ distribution summed over the $16 \eta_{c}$ decay modes. The data at $4.23,4.26$, and $4.36 \mathrm{GeV}$ were fitted simultaneously to the same signal function with common mass and width. Figure 3(a) shows the fitted results. The mass and width of $Z_{c}(4020)$ were measured to be $(4022.9 \pm 0.8 \pm 2.7) \mathrm{MeV} / c^{2}$ and $(7.9 \pm 2.7 \pm 2.6) \mathrm{MeV}$, respectively. The statistical significance of the $Z_{c}(4020)$ signal was found to be greater than $8.9 \sigma$.

Adding $Z_{c}(3900)$ with mass and width fixed to the BESIII measurements [5] to the fit results in a statistical significance of $2.1 \sigma$ (see the inset of Fig. 3(a)). At the $90 \%$ confidence level (C.L.), the upper limits on the production cross-sections are set to $\sigma\left(e^{+} e^{-} \rightarrow \pi^{ \pm} Z_{c}(3900)^{\mp} \rightarrow\right.$ $\left.\pi^{+} \pi^{-} h_{c}\right)<13 \mathrm{pb}$ at $4.23 \mathrm{GeV}$ and $<11 \mathrm{pb}$ at $4.26 \mathrm{GeV}$. These are lower than those of $Z_{c}(3900) \rightarrow \pi^{ \pm} J / \psi[5]$.

BESIII also observed $e^{+} e^{-} \rightarrow \pi^{0} \pi^{0} h_{c}$ at $\sqrt{s}=4.23$, 4.26 , and $4.36 \mathrm{GeV}$ for the first time [15]. The measured Born cross-sections were about half of those for $e^{+} e^{-} \rightarrow \pi^{+} \pi^{-} h_{c}$, which agree with expectations based on isospin symmetry within systematic uncertainties. A narrow structure with a mass of $(4023.9 \pm 2.2 \pm 3.8)$ $\mathrm{MeV} / c^{2}$ (for fitting, the width was fixed to that measured in the $e^{+} e^{-} \rightarrow \pi^{+} \pi^{-} h_{c}$ process [14] because of low statistics) was observed in the $\pi^{0} h_{c}$ mass spectrum (Fig. 3(b)). This structure is most likely the neutral isospin partner of the charged $Z_{c}(4020)$ observed in the $e^{+} e^{-} \rightarrow \pi^{+} \pi^{-} h_{c}$ process [14]. This observation indicates that there are no anomalously large isospin violations in $\pi \pi h_{c}$ and $\pi Z_{c}(4020)$ systems.

\subsubsection{Observation of $Z_{c}(4025)$}

The BESIII experiment also studied the $e^{+} e^{-} \rightarrow$ $\left(D^{*} \bar{D}^{*}\right)^{ \pm} \pi^{\mp}$ process at $4.26 \mathrm{GeV}$ using an $827 \mathrm{pb}^{-1}$ data sample [16]. Based on a partial reconstruction technique, the Born cross-section was measured to be $(137 \pm 9 \pm 15)$ pb. A structure near the $\left(D^{*} \bar{D}^{*}\right)^{ \pm}$threshold in the $\pi^{\mp}$ recoil mass spectrum was observed, and this is denoted as $Z_{c}(4025)$ (see Fig. 4(a)). The measured mass and width of the structure were $(4026.3 \pm 2.6 \pm 3.7) \mathrm{MeV} / c^{2}$ and
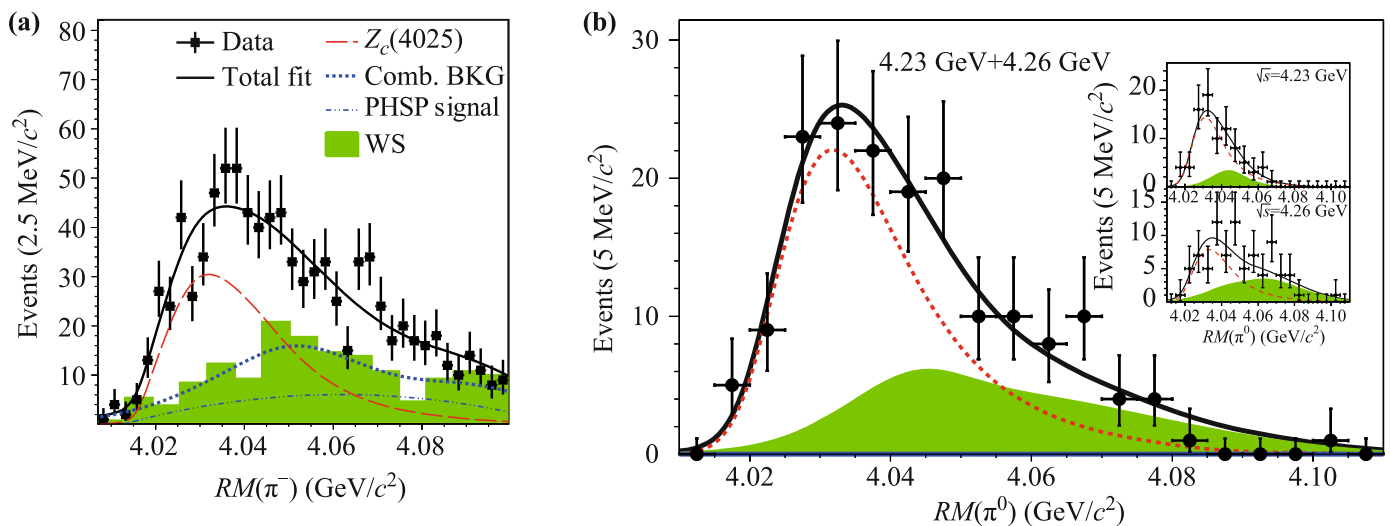

Fig. 4 Unbinned maximum likelihood fit to the $\pi^{\mp}$ recoil mass spectrum (a) in $e^{+} e^{-} \rightarrow\left(D^{*} \bar{D}^{*}\right)^{ \pm} \pi^{\mp}$ at $\sqrt{s}=4.26 \mathrm{GeV}$, and to the $\pi^{0}$ recoil mass spectrum (b) in $e^{+} e^{-} \rightarrow\left(D^{*} \bar{D}^{*}\right)^{0} \pi^{0}$ at $\sqrt{s}=4.23$ and $4.26 \mathrm{GeV}$ at BESIII. 
$(24.8 \pm 5.6 \pm 7.7) \mathrm{MeV}$, respectively, from the fit with a constant-width BW function for the signal. The associated production ratio $\frac{\sigma\left(e^{+} e^{-} \rightarrow Z_{c}^{ \pm}(4025) \pi^{\mp} \rightarrow\left(D^{*} \bar{D}^{*}\right)^{ \pm} \pi^{\mp}\right)}{\sigma\left(e^{+} e^{-} \rightarrow\left(D^{*} D^{*}\right)^{ \pm} \pi^{\mp}\right)}$ was determined to be $0.65 \pm 0.09 \pm 0.06$.

Using data at $\sqrt{s}=4.23$ and $4.26 \mathrm{GeV}$, a structure was observed in the $\pi^{0}$ recoil mass spectrum in the $e^{+} e^{-} \rightarrow D^{* 0} \bar{D}^{* 0}\left(D^{*+} D^{*-}\right) \pi^{0}$ process [17]. Assuming that the enhancement is due to a neutral state decaying to $D^{*} \bar{D}^{*}$, the mass and width of its pole position were determined to be $\left(4025.5_{-4.7}^{+2.0} \pm 3.1\right) \mathrm{MeV} / c^{2}$ and $\Gamma=$ $(23.0 \pm 6.0 \pm 1.0) \mathrm{MeV}$, respectively (see Fig. 4(b)). The Born cross-section $\sigma\left(e^{+} e^{-} \rightarrow Z_{c}(4025)^{0} \pi^{0} \rightarrow\left(D^{* 0} \bar{D}^{* 0}+\right.\right.$ $\left.\left.D^{*+} D^{*-}\right) \pi^{0}\right)$ was measured to be $(61.6 \pm 8.2 \pm 9.0) \mathrm{pb}$ at $4.23 \mathrm{GeV}$ and $(43.4 \pm 8.0 \pm 5.4) \mathrm{pb}$ at $4.26 \mathrm{GeV}$. The ratio $\frac{\sigma\left(e^{+} e^{-} \rightarrow Z_{c}(4025)^{0} \pi^{0} \rightarrow\left(D^{*} \bar{D}^{*}\right)^{0} \pi^{0}\right)}{\sigma\left(e^{+} e^{-} \rightarrow Z_{c}(4025)^{+} \pi^{-} \rightarrow\left(D^{*} \bar{D}^{*}\right)^{+} \pi^{-}\right)}$is compatible with unity at $\sqrt{s}=4.26 \mathrm{GeV}$, which is expected from isospin symmetry. In addition, $Z_{c}(4025)^{0}$ has a mass and width that are very close to those of $Z_{c}(4025)^{ \pm}$, which couples to $\left(D^{*} \bar{D}^{*}\right)^{ \pm}$. Therefore, the observed $Z_{c}(4025)^{0}$ state is a good candidate for the isospin partner of $Z_{c}(4025)^{ \pm}$.

As the $Z_{c}(4025)$ parameters agree to within $1.5 \sigma$ with those of $Z_{c}(4020)$, it is very probable that they are the same state. As the results for $Z_{c}(4025)^{ \pm}$are only from data at $4.26 \mathrm{GeV}$, extending the analysis to $4.23 \mathrm{GeV}$ and $4.36 \mathrm{GeV}$ will probably provide a definite answer.

\section{Observation of $Y(4260) \rightarrow \gamma X(3872)$}

BESIII observed $e^{+} e^{-} \rightarrow \gamma X(3872) \rightarrow \gamma \pi^{+} \pi^{-} J / \psi$, with $J / \psi$ reconstructed through its decays into lepton pairs $\left(\ell^{+} \ell^{-}=e^{+} e^{-}\right.$or $\left.\mu^{+} \mu^{-}\right)[18]$.

The $M\left(\pi^{+} \pi^{-} J / \psi\right)$ distribution (summed over all energy points), as shown in Fig. 5(a), was fitted to extract the mass and signal yield of $X(3872)$. The ISR $\psi(2 S)$ signal was used to calibrate the absolute mass scale and to extract the resolution difference between the data and a Monte Carlo (MC) simulation. Figure 5 shows the fitted result: the measured mass of $X(3872)$ was $(3871.9 \pm 0.7 \pm 0.2) \mathrm{MeV} / c^{2}$. From a fit with a floating width, we obtain a width of $\left(0.0_{-0.0}^{+1.7}\right) \mathrm{MeV}$, or less than $2.4 \mathrm{MeV}$ at the $90 \%$ C.L. The statistical significance of $X(3872)$ is $6.3 \sigma$.

The Born-order cross-section was measured, and the results are listed in Table 2. For 4.009 and $4.36 \mathrm{GeV}$ data, because the $X(3872)$ signal is not significant, upper limits on the production rates are given at the $90 \%$ C.L. The measured cross-sections at around $4.26 \mathrm{GeV}$ are an order of magnitude higher than the NRQCD calculation of continuum production [19], which may suggest the $X(3872)$ events come from resonance decays.

Table 2 The product of the Born cross section $\sigma^{B}\left(e^{+} e^{-} \rightarrow\right.$ $\gamma X(3872))$ and $\mathcal{B}\left(X(3872) \rightarrow \pi^{+} \pi^{-} J / \psi\right)$ at different energy points. The upper limits are given at $90 \%$ C.L.

\begin{tabular}{cc}
\hline$\sqrt{s}(\mathrm{GeV}) \sigma^{B}\left[e^{+} e^{-} \rightarrow \gamma X(3872)\right] \cdot \mathcal{B}\left(X(3872) \rightarrow \pi^{+} \pi^{-} J / \psi\right)(\mathrm{pb})$ \\
\hline 4.009 & $0.00 \pm 0.04 \pm 0.01$ or $<0.11$ \\
4.229 & $0.27 \pm 0.09 \pm 0.02$ \\
4.260 & $0.33 \pm 0.12 \pm 0.02$ \\
4.360 & $0.11 \pm 0.09 \pm 0.01$ or $<0.36$ \\
\hline
\end{tabular}

The energy-dependent cross-sections were fitted with a $Y(4260)$ resonance (parameters fixed to PDG [12] values), linear continuum, or E1-transition phase space $\left(\propto E_{\gamma}^{3}\right)$ term. Figure 5(b) shows all the fitted results, which imply that $\chi^{2} / \mathrm{ndf}=0.49 / 3($ C.L. $=92 \%), 5.5 / 2$ $($ C.L. $=6 \%)$, and $8.7 / 3($ C.L. $=3 \%)$ for a $Y(4260)$ resonance, linear continuum, and phase space distribution, respectively. Thus, the $Y(4260)$ resonance describes the data better than the other two options.

These observations strongly support the existence of the radiative transition process $Y(4260) \rightarrow \gamma X(3872)$. The $Y(4260) \rightarrow \gamma X(3872)$ process could be another previously unseen decay mode of the $Y(4260)$ resonance. Together with the transitions to the charged charmonium-
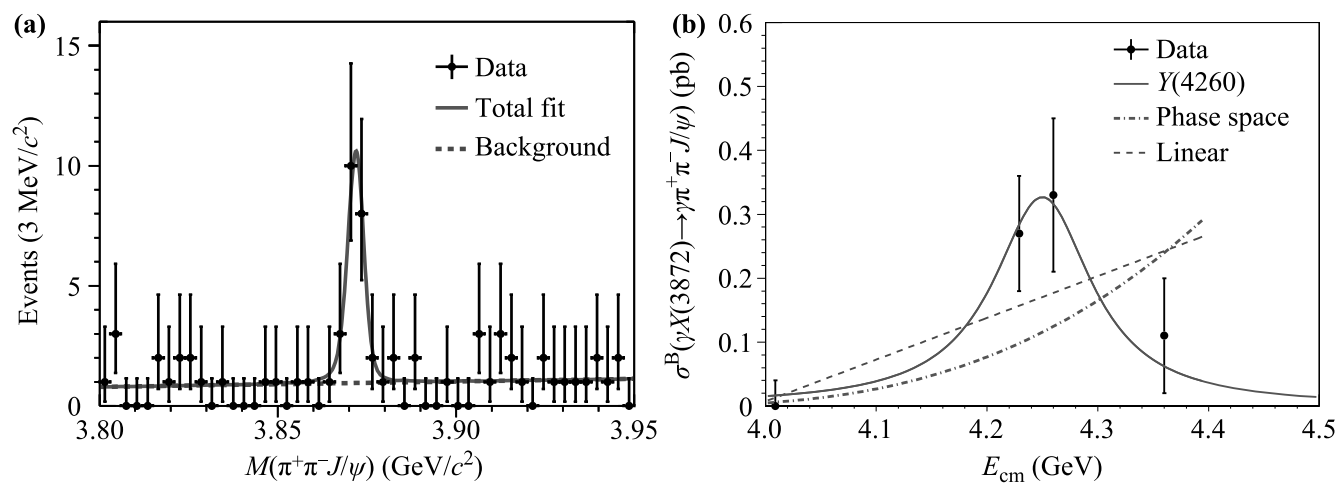

Fig. 5 (a) fit the $M\left(\pi^{+} \pi^{-} J / \psi\right)$ distribution observed at BESIII. Dots with error bars are data, the curves are the best fit. (b) fit to $\sigma^{B}\left[e^{+} e^{-} \rightarrow \gamma X(3872)\right] \times \mathcal{B}\left[X(3872) \rightarrow \pi^{+} \pi^{-} J / \psi\right]$ measured by BESIII with a $Y(4260)$ resonance (red solid curve), a linear continuum (blue dashed curve), or an E1-transition phase space term (red dotted-dashed curve). Dots with error bars are data. 

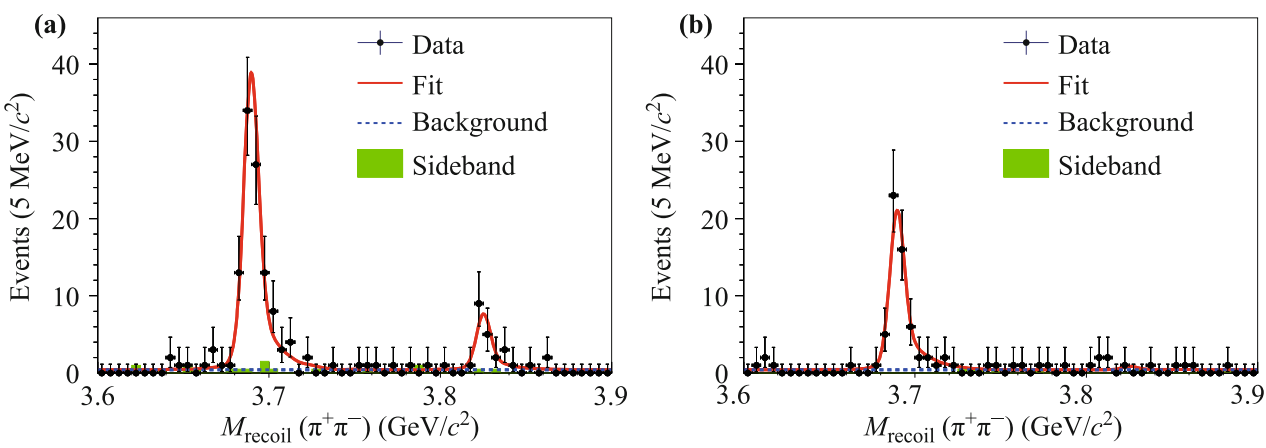

Fig. 6 Simultaneous fit to the $M_{\text {recoil }}\left(\pi^{+} \pi^{-}\right)$distribution of $\gamma \chi_{c 1}$ events (a) and $\gamma \chi_{c 2}$ events (b), respectively. Dots with error bars are data, red solid curves are total fit, dashed blue curves are background, and the green shaded histograms are $J / \psi$ mass sideband events.

like state $Z_{c}(3900)[5,8,9]$, this suggests that there might be some commonality in the nature of $X(3872), Y(4260)$, and $Z_{c}(3900)$, and so the model developed to interpret any one of them should also consider the other two. As an example, the authors of Ref. [20] integrated these states into a molecular picture to calculate $e^{+} e^{-} \rightarrow \gamma X(3872)$ cross-sections.

Combining the above with the $e^{+} e^{-} \rightarrow \pi^{+} \pi^{-} J / \psi$ cross-section measurement at $\sqrt{s}=4.26 \mathrm{GeV}$ from BESIII [5], we obtain $\sigma^{B}\left[e^{+} e^{-} \rightarrow \gamma X(3872)\right] \cdot \mathcal{B}[X(3872) \rightarrow$ $\left.\pi^{+} \pi^{-} J / \psi\right] / \sigma^{B}\left(e^{+} e^{-} \rightarrow \pi^{+} \pi^{-} J / \psi\right)=(5.2 \pm 1.9) \times$ $10^{-3}$, under the assumption that $X(3872)$ and $\pi^{+} \pi^{-} J / \psi$ are only produced from $Y(4260)$ decays. If we take $\mathcal{B}\left[X(3872) \rightarrow \pi^{+} \pi^{-} J / \psi\right]=5 \%[21]$, then $\mathcal{R}=$ $\frac{\mathcal{B}[Y(4260) \rightarrow \gamma X(3872)]}{\mathcal{B}\left(Y(4260) \rightarrow \pi^{+} \pi^{-} J / \psi\right)} \sim 0.1$.

\section{Observation of $\psi\left(1^{3} D_{2}\right)$}

BESIII observed $X(3823)$ in the $e^{+} e^{-} \rightarrow$ $\pi^{+} \pi^{-} X(3823) \rightarrow \pi^{+} \pi^{-} \gamma \chi_{c 1}$ process with a statistical significance of $6.2 \sigma$ in data samples at c.m. energies of $\sqrt{s}=4.23,4.26,4.36,4.42$, and $4.60 \mathrm{GeV}$ [22].

Figure 6 shows the fitted results to $\pi^{+} \pi^{-}$recoil mass distributions for events in the $\chi_{c 1}$ and $\chi_{c 2}$ signal regions. The fit yields $19 \pm 5 X(3823)$ signal events in the $\gamma \chi_{c 1}$ mode, with a measured mass of $X(3823)$ of $(3821.7 \pm 1.3 \pm 0.7) \mathrm{MeV} / c^{2}$, where the first error is statistical and the second systematic. For the $\gamma \chi_{c 2}$ mode, no significant $X(3823)$ signal was observed, and an upper limit on its production rate could be determined. The limited statistics do not allow a measurement of the intrinsic width of $X(3823)$. From a fit using the BW function (with a floating width parameter) convolved with Gaussian resolution, it can be determined that $\Gamma[X(3823)]<16 \mathrm{MeV}$ at the $90 \%$ C.L. (including systematic errors). This measurement agrees well with the values found by Belle [23].

The production cross-sections of $\sigma^{B}\left(e^{+} e^{-} \rightarrow\right.$ $\left.\pi^{+} \pi^{-} X(3823)\right) \cdot \mathcal{B}\left(X(3823) \rightarrow \gamma \chi_{c 1}, \gamma \chi_{c 2}\right)$ were also measured at these c.m. energies. The cross-sections of $e^{+} e^{-} \rightarrow \pi^{+} \pi^{-} X(3823)$ were fitted with the $Y(4360)$ shape or the $\psi(4415)$ shape, with their resonance parameters fixed to the PDG values [12]. Figure 7 shows the fitted results, which give the Kolmogorov-Smirnov statistic $D_{5, \mathrm{obs}}^{\mathrm{H} 1}=0.151$ for the $Y(4360)$ hypothesis $(\mathrm{H} 1)$ and $D_{5, \mathrm{obs}}^{\mathrm{H} 2}=0.169$ for the $\psi(4415)$ hypothesis $(\mathrm{H} 2)$, based on the Kolmogorov-Smirnov test [24]. Thus, both the $Y(4360)$ and $\psi(4415)$ hypotheses $\left(D_{5, \mathrm{obs}}^{\mathrm{H} 1}, D_{5, \mathrm{obs}}^{\mathrm{H} 2}<\right.$ $\left.D_{5,0.1}=0.509\right)$ are accepted at a $90 \%$ C.L.

The $X(3823)$ resonance is a good candidate for the $\psi\left(1^{3} D_{2}\right)$ charmonium state. According to potential models [25], the $D$-wave charmonium states are expected to be within a mass range of $3.82-3.85 \mathrm{GeV}$. Among these, the $1^{1} D_{2} \rightarrow \gamma \chi_{c 1}$ transition is forbidden because of $\mathrm{C}$ parity conservation, and the amplitude for $1^{3} D_{3} \rightarrow \gamma \chi_{c 1}$ is expected to be small [26]. The mass of $\psi\left(1^{3} D_{2}\right)$ is in the $3.810 \sim 3.840 \mathrm{GeV} / c^{2}$ range predicted by several phenomenological calculations [27-32]. In this case, the mass of $\psi\left(1^{3} D_{2}\right)$ was above the $D \bar{D}$ threshold but below the $D \bar{D}^{*}$ threshold. Because $\psi\left(1^{3} D_{2}\right) \rightarrow D \bar{D}$ violates parity, $\psi\left(1^{3} D_{2}\right)$ is expected to be narrow, in agreement

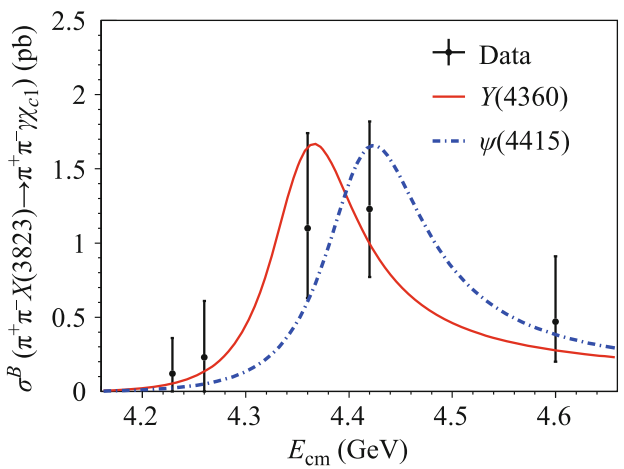

Fig. 7 Comparison of the energy-dependent cross sections of $\sigma^{B}\left[e^{+} e^{-} \rightarrow \pi^{+} \pi^{-} X(3823)\right] \cdot \mathcal{B}\left(X(3823) \rightarrow \gamma \chi_{c 1}\right)$ to the $Y(4360)$ and $\psi(4415)$ line shapes. Dots with error bars (statistical only) are data. The red solid (blue dashed) curve shows a fit with the $Y(4360)(\psi(4415))$ line shape. 
with the observation, and $\psi\left(1^{3} D_{2}\right) \rightarrow \gamma \chi_{c 1}$ is expected to be a dominant decay mode [27-33]. From the cross-section measurement, we obtain the ratio $\frac{\mathcal{B}\left[X(3823) \rightarrow \gamma \chi_{c 2}\right]}{\mathcal{B}\left[X(3823) \rightarrow \gamma \chi_{c 1}\right]}<0.42$ (where systematic uncertainty cancels) at the $90 \%$ C.L., which also agrees with expectations for the $\psi\left(1^{3} D_{2}\right)$ state [33].

\section{Search for $e^{+} e^{-} \rightarrow \gamma \chi_{c J}$ and $e^{+} e^{-} \rightarrow$ $\gamma Y(4140)$}

Using data samples collected at $\sqrt{s}=4.009,4.23,4.26$, and $4.36 \mathrm{GeV}$, BESIII searched for $e^{+} e^{-} \rightarrow \gamma \chi_{c J}(J=$ $0,1,2)$ with the subsequent decay $\chi_{c J} \rightarrow \gamma J / \psi$ and $J / \psi \rightarrow \mu^{+} \mu^{-}$. Evidence for the $e^{+} e^{-} \rightarrow \gamma \chi_{c 1}$ and $e^{+} e^{-} \rightarrow \gamma \chi_{c 2}$ processes was observed with a statistical significance of $3.0 \sigma$ and $3.4 \sigma$, respectively [34]. No evidence for $e^{+} e^{-} \rightarrow \gamma \chi_{c 0}$ was observed. The corresponding Born cross-sections of $e^{+} e^{-} \rightarrow \gamma \chi_{c J}$ at different c.m. energies were calculated. Under the assumption that $\chi_{c J}$ signals were absent, the upper limits on the Born cross-sections were calculated at the $90 \%$ C.L. These upper limits on the Born cross-section of $e^{+} e^{-} \rightarrow \gamma \chi_{c J}$ are compatible with the theoretical prediction from the NRQCD calculation [19].

BESIII searched for $Y(4140)$ via $e^{+} e^{-} \rightarrow \gamma \phi J / \psi$ at $\sqrt{s}=4.23,4.26$, and $4.36 \mathrm{GeV}$, but no significant $Y(4140)$ signal was observed in any of the data samples [35]. The upper limits of the product of the crosssection and branching fraction $\sigma\left[e^{+} e^{-} \rightarrow \gamma Y(4140)\right]$. $\mathcal{B}(Y(4140) \rightarrow \phi J / \psi)$ at the $90 \%$ C.L. were estimated to be $0.35,0.28$, and $0.33 \mathrm{pb}$ at $\sqrt{s}=4.23,4.26$, and 4.36 $\mathrm{GeV}$, respectively.

These upper limits can be compared with the $X(3872)$ production rates [18], which were measured from the same data samples (see Section 3). The latter were $\sigma\left[e^{+} e^{-} \rightarrow \gamma X(3872)\right] \cdot \mathcal{B}\left(X(3872) \rightarrow \pi^{+} \pi^{-} J / \psi\right)=$ $[0.27 \pm 0.09$ (stat) \pm 0.02 (syst) $] \mathrm{pb},[0.33 \pm 0.12$ (stat) \pm 0.02 (syst) $] \mathrm{pb}$, and $[0.11 \pm 0.09$ (stat) \pm 0.01 (syst) $] \mathrm{pb}$ at $\sqrt{s}=4.23,4.26$, and $4.36 \mathrm{GeV}$, respectively, which are of the same order of magnitude as the upper limits of $\sigma\left[e^{+} e^{-} \rightarrow \gamma Y(4140)\right] \cdot \mathcal{B}(Y(4140) \rightarrow \phi J / \psi)$ at the same energy.

The branching fraction $\mathcal{B}(Y(4140) \rightarrow \phi J / \psi)$ has not previously been measured. Using the partial width of $Y(4140) \rightarrow \phi J / \psi$ calculated under the molecule hypothesis [36] and the total width of the $Y(4140)$ measured by CDF [37], the branching fraction was estimated to be approximately $30 \%$. A rough estimation for $\mathcal{B}(X(3872) \rightarrow$ $\left.\pi^{+} \pi^{-} J / \psi\right)$ is $5 \%$ [21]. Combining these numbers, the ratio $\sigma\left[e^{+} e^{-} \rightarrow \gamma Y(4140)\right] / \sigma\left[e^{+} e^{-} \rightarrow \gamma X(3872)\right]$ can be estimated to be of the order of 0.1 or less at $\sqrt{s}=4.23$ and $4.26 \mathrm{GeV}$.

\section{Structures in $e^{+} e^{-} \rightarrow$ charmonium + hadrons}

\subsection{Observation of $e^{+} e^{-} \rightarrow \omega \chi_{c 0}$}

Based on data samples collected between $\sqrt{s}=4.21$ and $4.42 \mathrm{GeV}$, the $e^{+} e^{-} \rightarrow \omega \chi_{c 0}$ process was observed at $\sqrt{s}=4.23$ and $4.26 \mathrm{GeV}$ for the first time [38], and the Born cross-sections were measured to be $(55.4 \pm 6.0 \pm 5.9)$ and $(23.7 \pm 5.3 \pm 3.5) \mathrm{pb}$, respectively. For other energy points, no significant signals were found, and upper limits on the cross-section at the $90 \%$ C.L. were determined.

The data reveal a sizable $\omega \chi_{c 0}$ production at around $4.23 \mathrm{GeV} / c^{2}$, as predicted in Ref. [39]. By assuming the $\omega \chi_{c 0}$ signals come from a single resonance, the $\Gamma_{e e} \mathcal{B}\left(\omega \chi_{c 0}\right)$, mass, and width of the resonance were determined to be $(2.7 \pm 0.5 \pm 0.4) \mathrm{eV},(4230 \pm 8 \pm 6) \mathrm{MeV} / c^{2}$, and $(38 \pm 12 \pm 2) \mathrm{MeV}$, respectively (the fit is shown in Fig. 8). The parameters are consistent with those of the narrow structure in the $e^{+} e^{-} \rightarrow \pi^{+} \pi^{-} h_{c}$ process [40], but are not consistent with those obtained by fitting a single resonance to the $\pi^{+} \pi^{-} J / \psi$ cross-section [41] (with a mass of $\left(4259 \pm 8_{-6}^{+2}\right) \mathrm{MeV} / c^{2}$ and a width of $\left.\left(88 \pm 23_{-4}^{+6}\right) \mathrm{MeV}\right)$. This suggests that the observed $\omega \chi_{c 0}$ signals are unlikely to originate from $Y(4260)$.

The $e^{+} e^{-} \rightarrow \omega \chi_{c 1,2}$ channels were also sought, but no significant signals were observed; upper limits at the $90 \%$ C.L. on the production cross-sections were determined. The very small measured ratios of $e^{+} e^{-} \rightarrow \omega \chi_{c 1,2}$ crosssections to those for $e^{+} e^{-} \rightarrow \omega \chi_{c 0}$ are inconsistent with the prediction in Ref. [42].

\subsection{Measurement of $e^{+} e^{-} \rightarrow \eta J / \psi$}

Using data samples collected at energies of 3.81-4.60

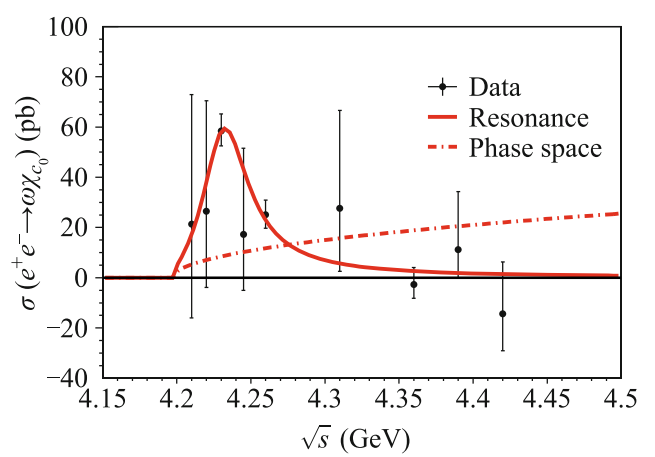

Fig. 8 Fit to $\sigma\left(e^{+} e^{-} \rightarrow \omega \chi_{c 0}\right)$ with a resonance (solid curve), or a phase space term (dot-dashed curve). Dots with error bars are the dressed cross sections. The uncertainties are statistical only. 

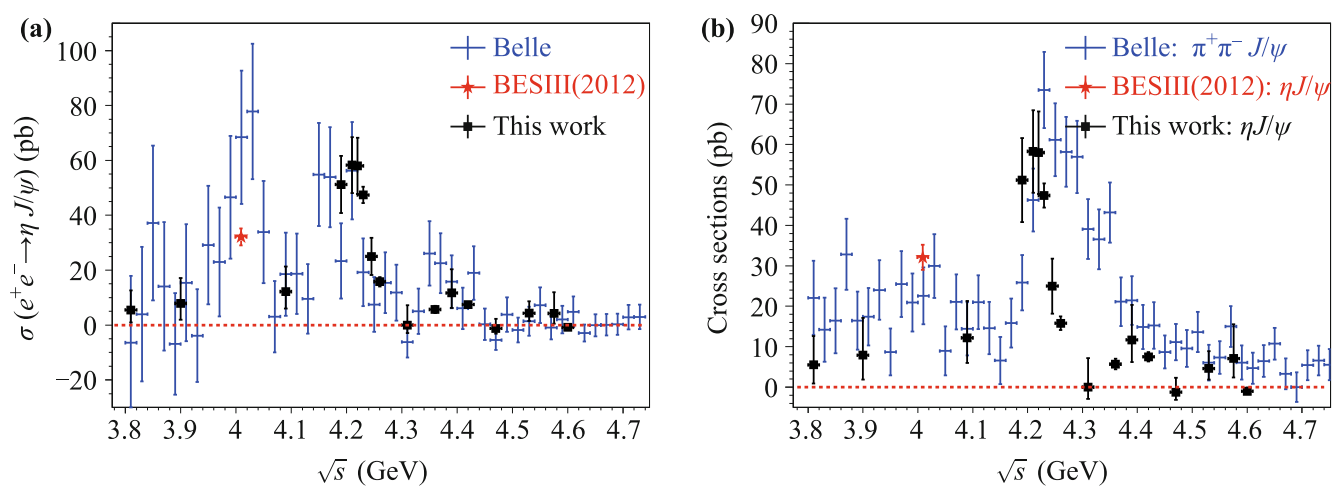

Fig. 9 A comparison of the measured Born cross sections of $e^{+} e^{-} \rightarrow \eta J / \psi$ to those of the previous measurements [13, 44] (a), and to those of $e^{+} e^{-} \rightarrow \pi^{+} \pi^{-} J / \psi$ from Belle [8]. In these two plots, the black square dots and the red star dots are the results of $\eta J / \psi$ obtained from BESIII. The blue dots are results of $\eta J / \psi(\mathbf{a})$ and $\pi^{+} \pi^{-} J / \psi$ (b) from Belle. The errors are statistical only for Belle's results, and are final combined uncertainties for BESIII's results.

$\mathrm{GeV}, \mathrm{BESIII}$ analyzed $e^{+} e^{-} \rightarrow \eta J / \psi[43]$. Statistically significant $\eta$ signals were observed, and the corresponding Born cross-sections were measured. In addition, a search for the $e^{+} e^{-} \rightarrow \pi^{0} J / \psi$ process observed no significant signals, and upper limits at the $90 \%$ C.L. on the Born cross-section were set.

A comparison of the Born cross-sections $\sigma\left(e^{+} e^{-} \rightarrow\right.$ $\eta J / \psi)$ in this measurement to previous results $[13,44]$ is shown in Fig. 9(a), indicating very good agreement. The measured Born cross-sections were also compared to those of $e^{+} e^{-} \rightarrow \pi^{+} \pi^{-} J / \psi$ obtained from the Belle experiment [8], as shown in Fig. 9(b). Different line shapes can be observed in these two processes, indicating that the production mechanism of $\eta J / \psi$ differs from that of $\pi^{+} \pi^{-} J / \psi$ in the vicinity of $\sqrt{s}=4.1-4.6 \mathrm{GeV}$. This could indicate the existence of a rich spectrum of the $Y$ states in this energy region with different coupling strengths to various decay modes.

\section{More data for $X Y Z$ study}

As shown in previous sections, BESIII has achieved a lot in the study of the $X Y Z$ states and the conventional charmonium states. However, there are more questions to be answered with the currently available data and, more importantly, with the data samples that BESIII is able to collect in the next few years.

A few topics that need to be studied with more data are listed below.

- In the $X$ sector:

- Where are the $X(3872)$ and $\psi\left(1{ }^{3} D_{2}\right)$ coming from, resonance decays or continuum production?

- Can the other $X$ states, such as XYZ(3940),
$X(3915), X(4140)(Y(4140))$, and $X(4350)$ be produced in a similar way?

- Can the charmonium 2P, 3P states and the Swave spin-singlet states $(3 \mathrm{~S}, 4 \mathrm{~S}, 5 \mathrm{~S})$ be observed in radiative transitions?

- In the $Y / \psi$ sector:

- Is the $Y(4260)$ structure a single resonance, or does it have a more complicated sub-structure? Is $Y(4008)$ a real resonance?

- What are the other decay modes of $Y(4360)$ ?

- What is hidden in the $e^{+} e^{-} \rightarrow \pi^{+} \pi^{-} h_{c}$ line shape?

- Is the $Y$ (4660) observed in $e^{+} e^{-} \rightarrow \pi^{+} \pi^{-} \psi(2 S)$ the same as the $Y(4630)$ observed in $e^{+} e^{-} \rightarrow$ $\Lambda_{c}^{+} \Lambda_{c}^{-}[45]$ ?

- What is the correlation between charm production ( $e^{+} e^{-} \rightarrow$ open charm final states) and charmonium production?

- Where is the vector charmonium $3 D$ state?

- Are there charmonium states between $\psi(4160)$ and $\psi(4415)$, and/or between $\psi(4415)$ and $Y(4660) ?$

- Can the vector charmonium hybrid state be observed $[46,47]$ ?

- In the $Z$ sector:

- Are the $Z_{c}$ states produced from resonance decays or from continuum production?

- Is there a $Z_{c s}$ state decaying into $K^{ \pm} J / \psi$ or $D_{s}^{-} D^{* 0}+$ c.c., $D_{s}^{*-} D^{0}+$ c.c.?

- Are there more $Z_{c}$ and $Z_{c s}$ states?

- In the $C$ sector:

- Can $D_{s 0}(2317)$ be produced and studied?

- Can the other excited charmed mesons be produced and studied with high-energy data? 
BESIII is going to collect about $3 \mathrm{fb}^{-1}$ of data in the vicinity of $\psi(4160)$ to study the $D_{s}$ decay properties in 2015-2016 run. These data can be used to answer some of the questions listed above. A few specific topics are listed here.

1) In $e^{+} e^{-} \rightarrow \pi^{+} \pi^{-} J / \psi$, there is a dip at around 4.17 $\mathrm{GeV}$ and a sharp increase at around $4.23 \mathrm{GeV}[8]$. The data may help to identify where the turning point is located.

2) For the $e^{+} e^{-} \rightarrow \omega \chi_{c 0}$ process [38], there are few data points close to the threshold to support the claim of a narrow structure.

3) For the $e^{+} e^{-} \rightarrow K^{+} K^{-} J / \psi$ process, it seems there is a structure at around $4.2 \mathrm{GeV}[48]$.

4) In the $e^{+} e^{-} \rightarrow \eta J / \psi$ process, the line shape differs from that of $e^{+} e^{-} \rightarrow \pi^{+} \pi^{-} J / \psi$, with a peak at around $4.2 \mathrm{GeV}$, but there are no data points on the left side.

5) In $e^{+} e^{-} \rightarrow \eta h_{c}$, is there a structure close to the threshold? What does the $e^{+} e^{-} \rightarrow \eta^{\prime} J / \psi$ line shape look like close to the threshold?

6) Are the $e^{+} e^{-} \rightarrow \gamma \chi_{c J}$ signals from $\psi(4160)$ or $Y(4260)$, or something else [34]?

7) The search for $F$-wave charmonium states via $\psi(4160)$ decays.

8) The search for $\gamma \chi_{c}(2 P), \gamma \mathrm{XYZ}(3940), \gamma X(3915)$ from $\psi(4160)$ decays.

More data are always better, but as we need to collect data at many points and the collection period is limited, we need to define lower limits for the number of data points and the luminosity needed at each point to ensure meaningful measurements of the physical quantities of interest.

As we require high-precision cross-sections for all of the open charm modes and the cross-sections of some hadronic transition modes to fit the resonant parameters of the vector states, we require sufficient data points to give the excitation curve of any of the existing vector resonances and possible hidden structures in the full energy range.

Some special issues need to be considered: (i) the thresholds of all the open charm and charmonium + hadron final states; (ii) possible energy regions where large interference effects are expected; (iii) energy regions where narrow structures are expected; (iv) energy regions that were not well explored before; and (v) the beam energy spread.

From previous calculations and measurements, it is known that the energy spread of BEPCII at energy ranges of $3.8-5.0 \mathrm{GeV}$ is around $1.4-2.0 \mathrm{MeV}$. In this case, we would not use energy steps finer than three times the energy spread. That is, unless very necessary, we would not take data at energy points less than $5 \mathrm{MeV}$ from an existing data point. Thus, we would take data with $5 \mathrm{MeV}$ steps in the energy regions where narrow structures or dramatic effects are expected, such as the thresholds for open charm or hidden charm final states, and the low mass shoulder of $Y(4260)$, where interference effects have been reported [8]. Otherwise, $10 \mathrm{MeV}$ steps will be used.

The limit on the data size is set according to the precision of the hadronic transition modes, which typically have cross-sections of a few to a few tens of picobarns. From the analyses in previous sections, we find that an integrated luminosity of $500 \mathrm{pb}^{-1}$ is needed to reach a reasonably high precision for most modes of interest. To search for the $X Y Z$ and charmonium states via radiative transition, a data sample of at least $500 \mathrm{pb}^{-1}$ is also needed to reach a $5 \sigma$ level observation of a signal if the production rate is $1 \times 10^{-4}$ or higher. A detailed MC study of the precision that can be reached or the exact luminosity needed for each energy point is necessary, as the background level may be very different at different resonant peaks.

With the above principles in mind, we propose to collect data samples at about 60 c.m. energies from $4.0 \mathrm{GeV}$ to the maximum energy that BEPCII can reach (currently $4.6 \mathrm{GeV}$ ) in $10 \mathrm{MeV}$ steps. Around $500 \mathrm{pb}^{-1}$ at each energy will be necessary for a comprehensive study of the $X Y Z$ and charmonium states. As BESIII has already accumulated about $5 \mathrm{fb}^{-1}$ (see Table 1), another $25 \mathrm{fb}^{-1}$ data should be accumulated. This will take about 5 years at BEPCII [4].

\section{$8 \quad X Y Z$ study in future experiments}

Belle-II [49] will start collecting data in 2018, and will accumulate $50 \mathrm{ab}^{-1}$ data at the $\Upsilon(4 S)$ peak by 2024 . These data samples can be used to study the XYZ and charmonium states in many different ways [1], among which ISR can produce events in the same energy range covered by BESIII. Figure 10 shows the effective luminosity at BEPCII energy in the Belle-II data samples. We can see that, for $10 \mathrm{ab}^{-1}$ Belle-II data, we have about 400$500 \mathrm{pb}^{-1}$ data for every $10 \mathrm{MeV}$ in the range $4-5 \mathrm{GeV}$, comparable to the data sample proposed at BESIII in the previous section. Of course, the ISR analyses have a lower efficiency than in direct $e^{+} e^{-}$collisions because of the extra ISR photons and the boost given to events along the beam direction. Even taking these effects into 


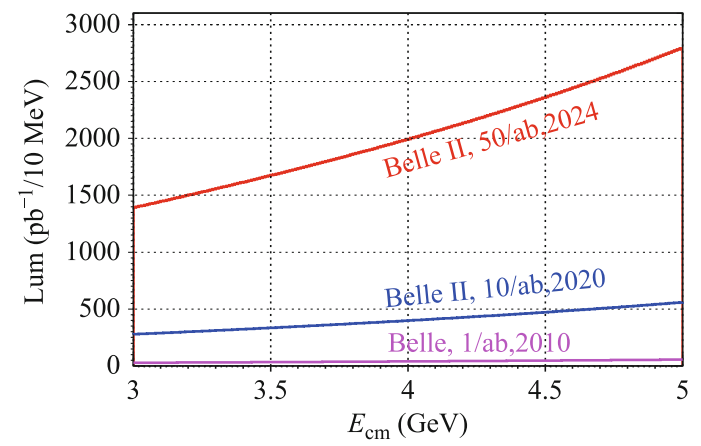

Fig. 10 Effective luminosity at low energy in the Belle and BelleII $\Upsilon(4 S)$ data samples.

account, the full Belle-II data sample, which corresponds to about $2000-2300 \mathrm{pb}^{-1}$ data for every $10 \mathrm{MeV}$ from 4-5 GeV, will result in similar statistics for modes like $e^{+} e^{-} \rightarrow \pi^{+} \pi^{-} J / \psi$. Belle-II has the advantage that data at different energies will be accumulated at the same time, making the analysis much simpler than at BESIII at 60 data points. In addition, Belle-II can produce events above $4.6 \mathrm{GeV}$, which is currently the maximum energy of BEPCII. Possible upgrades to increase the maximum c.m. energy of BEPCII will obviously expand the physical possibilities of BESIII.

The HIEPA project [50] being discussed at this workshop will improve the aforementioned studies in many aspects, particularly with c.m. energies of up to $7 \mathrm{GeV}$ and luminosity improvements of a factor of 100 . These will allow a finer scan in the full energy region with more integrated luminosity. This will enable a better understanding of all the studies listed in this article.

\section{Conclusion}

With the world's largest data samples at energies of 3.8-4.6 GeV, the BESIII experiment made a significant contribution to the study of the charmonium and $X Y Z$ states. To further strengthen such studies, BESIII may collect more data from 4.0-4.6 GeV (or even higher, if possible). These data will be complementary to the BelleII study, with many other production mechanisms. The HIEPA project may enable a systematic understanding of the nature of the $X Y Z$ and charmonium states.

Acknowledgements This work was supported in part by the National Natural Science Foundation of China (NSFC) under Grant Nos. 11235011 and 11475187; the Ministry of Science and Technology of China under Grant No. 2015CB856701, and the CAS Center for Excellence in Particle Physics (CCEPP).

Open Access This article is distributed under the terms of the Creative Commons Attribution License which permits any use, distribution, and reproduction in any medium, provided the original author(s) and the source are credited.

\section{References and notes}

1. A. J. Bevan, et al. (BaBar and Belle Collaborations), The physics of the $B$ factories, Eur. Phys. J. C 74, 3026 (2014)

2. For a recent review, see: N. Brambilla, et al., Heavy quarkonium: progress, puzzles, and opportunities, Eur. Phys. J. C 71, 1534 (2011)

3. M. Ablikim, et al. (BESIII Collaboration), Design and construction of the BESIII detector, Nucl. Instrum. Methods Phys. Res. Sect. A 614, 345 (2010)

4. D. M. Asner, et al., Physics at BES-III, Int. J. Mod. Phys. A 24, S1 (2009)

5. M. Ablikim, et al. (BESIII Collaboration), Observation of a charged charmoniumlike structure in $e^{+} e^{-} \rightarrow \pi^{+} \pi^{-} J / \psi$ at $\sqrt{s}=4.26 \mathrm{GeV}$, Phys. Rev. Lett. 110, 252001 (2013)

6. M. Ablikim, et al. (BESIII Collaboration), Measurements of the center-of-mass energies via the di-muon process at BESIII, to be submitted to Chin. Phys. C

7. M. Ablikim, et al. (BESIII Collaboration), Precision measurement of the integrated luminosity of the data taken by BESIII at center of mass energies between $3.810 \mathrm{GeV}$ and $4.600 \mathrm{GeV}$, arXiv: 1503.03408 [hep-ex]

8. Z. Q. Liu, et al. (Belle Collaboration), Study of $e^{+} e^{-} \rightarrow$ $\pi^{+} \pi^{-} J / \psi$ and observation of a charged charmoniumlike state at Belle, Phys. Rev. Lett. 110, 252002 (2013)

9. T. Xiao, S. Dobbs, A. Tomaradze, and K. K. Seth, Observation of the charged hadron $Z_{c}^{ \pm}(3900)$ and evidence for the neutral $Z_{c}^{0}(3900)$ in $e^{+} e^{-} \rightarrow \pi \pi J / \psi$ at $\sqrt{s}=4170 \mathrm{MeV}$, Phys. Lett. $B$ 727, 366 (2013)

10. M. Ablikim, et al. (BESIII Collaboration), Observation of $Z_{c}^{0}(3900)$ in $e^{+} e^{-} \rightarrow \pi^{0} \pi^{0} J / \psi$, Phys. Rev. Lett. 110, 252001 (2013)

11. M. Ablikim, et al. (BESIII Collaboration), Observation of a charged $\left(D \bar{D}^{*}\right)^{-}$mass peak in $e^{+} e^{-} \rightarrow \pi^{+}\left(D \bar{D}^{*}\right)^{-}$at $E_{c m}=4.26 \mathrm{GeV}$, Phys. Rev. Lett. 112, 022001 (2014)

12. K. A. Olive, et al. (Particle Data Group), The Review of Particle Physics, Chin. Phys. C 38, 090001 (2014)

13. M. Ablikim, et al. (BESIII Collaboration), Observation of $e^{+} e^{-} \rightarrow \eta J / \psi$ at center-of-mass energy $\sqrt{s}=4.009 \mathrm{GeV}$, Phys. Rev. D 86, 071101 (2012)

14. M. Ablikim, et al. (BESIII Collaboration), Observation of a charged charmoniumlike structure $Z_{c}(4020)$ and search for the $Z_{c}(3900)$ in $e^{+} e^{-} \rightarrow \pi^{+} \pi^{-} h_{c}$, Phys. Rev. Lett. 111, 242001 (2013)

15. M. Ablikim, et al. (BESIII Collaboration), Observation of $e^{+} e^{-} \rightarrow \pi^{0} \pi^{0} h_{c}$ and a neutral charmoniumlike structure $Z_{c}(4020)^{0}$, Phys. Rev. Lett. 113(21), 212002 (2014)

16. M. Ablikim, et al. (BESIII Collaboration), Observation of a charged charmoniumlike structure in $e^{+} e^{-} \rightarrow\left(D^{*} \bar{D}^{*}\right)^{ \pm} \pi^{\mp}$ at $\sqrt{s}=4.26 \mathrm{GeV}$, Phys. Rev. Lett. 112, 132001 (2014)

17. M. Ablikim, et al. (BESIII Collaboration), Observation of a neutral charmoniumlike state $Z_{c}(4025)^{0}$ in $e^{+} e^{-} \rightarrow$ $\left(D^{*} \bar{D}^{*}\right)^{0} \pi^{0}$, arXiv: 1507.02404 [hep-ex] 
18. M. Ablikim, et al. (BESIII Collaboration), Observation of $e^{+} e^{-} \rightarrow \gamma X(3872)$ at BESIII, Phys. Rev. Lett. 112(9), 092001 (2014)

19. K. T. Chao, Z. G. He, D. Li, and C. Meng, Search for $C=+$ charmonium states in $e^{+} e^{-} \rightarrow \gamma+X$ at BEPCII/BESIII, arXiv: 1310.8597 [hep-ph]

20. F. K. Guo, C. Hanhart, U. G. Meißner, Q. Wang, and Q. Zhao, Production of the $X(3872)$ in charmonia radiative decays, Phys. Lett. B 725, 127 (2013)

21. C. Z. Yuan (for the BES and Belle Collaborations), Exotic Hadrons, arXiv: 0910.3138 [hep-ex]. We take 5\% from the range presented in the paper of $2.3 \%<\mathcal{B}(X(3872) \rightarrow$ $\left.\pi^{+} \pi^{-} J / \psi\right)<6.6 \%$ at $90 \%$ C.L.

22. M. Ablikim, et al. (BESIII Collaboration), Observation of the $\psi\left(1^{3} D_{2}\right)$ state in $e^{+} e^{-} \rightarrow \pi^{+} \pi^{-} \gamma \chi_{c 1}$ at BESIII, Phys. Rev. Lett. 115(1), 011803 (2015)

23. V. Bhardwaj, et al. (Belle Collaboration), Evidence of a new narrow resonance decaying to $\chi_{c 1} \gamma$ in $B \rightarrow \chi_{c 1} \gamma K$, Phys. Rev. Lett. 111(3), 032001 (2013)

24. A. Kolmogorov, Sulla determinazione empirica di una legge di distribuzione, G. Inst. Ital. Attuari. 4, 83 (1933)

25. E. Eichten, K. Gottfried, T. Kinoshita, K. D. Lane, and T. M. Yan, Charmonium: The Model, Phys. Rev. D 17, 3090 (1978) [Phys. Rev. D 21, 313 (1980)]

26. T. Barnes, S. Godfrey, and E. S. Swanson, Higher charmonia, Phys. Rev. D 72, 054026 (2005)

27. S. Godfrey and N. Isgur, Mesons in a relativized quark model with chromodynamics, Phys. Rev. D 32, 189 (1985)

28. W. Kwong, J. L. Rosner, and C. Quigg, Heavy quark systems, Ann. Rev. Nucl. Part. Sci. 37, 325 (1987)

29. D. Ebert, R. N. Faustov, and V. O. Galkin, Properties of heavy quarkonia and $B_{c}$ mesons in the relativistic quark model, Phys. Rev. D 67, 014027 (2003)

30. E. J. Eichten, K. Lane, and C. Quigg, Charmonium levels near threshold and the narrow state $X(3872) \rightarrow \pi^{+} \pi^{-} J / \psi$, Phys. Rev. D 69, 094019 (2004)

31. B. Q. Li and K. T. Chao, Higher charmonia and X,Y,Z states with screened potential, Phys. Rev. D 79, 094004 (2009)

32. M. Blank and A. Krassnigg, Bottomonium in a BetheSalpeter-equation study, Phys. Rev. D 84, 096014 (2011)

33. C. F. Qiao, F. Yuan, and K. T. Chao, A Crucial test for color octet production mechanism in $Z^{0}$ decays, Phys. Rev. D 55, 4001 (1997)

34. M. Ablikim, et al. (BESIII Collaboration), Evidence for $e^{+} e^{-} \rightarrow \gamma \chi_{c 1,2}$ at center-of-mass energies from 4.009 to $4.360 \mathrm{GeV}$, Chin. Phys. C 39(4), 041001 (2015)

35. M. Ablikim, et al. (BESIII Collaboration), Search for the $Y(4140)$ via $e^{+} e^{-} \rightarrow \gamma J / \psi$ at $\sqrt{s}=4.23,4.26$, and $4.36 \mathrm{GeV}$, Phys. Rev. D 91(3), 032002 (2015)
36. T. Branz, T. Gutsche, and V. E. Lyubovitskij, Hadronic molecule structure of the $Y(3940)$ and $Y(4140)$, Phys. Rev. D 80, 054019 (2009)

37. T. Aaltonen, et al. (CDF Collaboration), Observation of the $Y(4140)$ structure in the $J / \psi \phi$ mass spectrum in $B^{ \pm} \rightarrow$ $J / \psi \phi K$ decays, arXiv: 1101.6058 [hep-ex]

38. M. Ablikim, et al. (BESIII Collaboration), Study of $e^{+} e^{-} \rightarrow$ $\omega \chi_{c J}$ at center-of-mass energies from 4.21 to $4.42 \mathrm{GeV}$, Phys. Rev. Lett. 114(9), 092003 (2015)

39. L. Y. Dai, M. Shi, G. Y. Tang, and H. Q. Zheng, Nature of X(4260), Phys. Rev. D 92(1), 014020 (2015)

40. Chang-Zheng Yuan, Evidence for resonant structures in $e^{+} e^{-} \rightarrow \pi^{+} \pi^{-} h_{c}$, Chin. Phys. C 38, 043001 (2014)

41. B. Aubert, et al. (BaBar Collaboration), Observation of a broad structure in the $\pi^{+} \pi^{-} J / \psi$ mass spectrum around 4.26-GeV/c² Phys. Rev. Lett. 95, 142001 (2005)

42. L. Ma, X. H. Liu, X. Liu, and S. L. Zhu, Strong decays of the XYZ states, Phys. Rev. D 91(3), 034032 (2015)

43. M. Ablikim, et al. (BESIII Collaboration), Measurement of the $e^{+} e^{-} \rightarrow \eta J / \psi$ cross section and search for $e^{+} e^{-} \rightarrow$ $\pi^{0} \mathrm{~J} / \psi$ at center-of-mass energies between 3.810 and 4.600 GeV, Phys. Rev. D 91(11), 112005 (2015)

44. X. L. Wang, et al. (Belle Collaboration), Observation of $\psi(4040)$ and $\psi(4160)$ decay into $\eta J / \psi$, Phys. Rev. D 87(5), 051101 (2013)

45. G. Pakhlova, et al. (Belle Collaboration), Observation of a near-threshold enhancement in the $e^{+} e^{-} \rightarrow \Lambda_{c}^{+} \Lambda_{c}^{-}$cross section using initial-state radiation, Phys. Rev. Lett. 101, 172001 (2008)

46. P. Guo, A. P. Szczepaniak, G. Galata, A. Vassallo, and E. Santopinto, Heavy quarkonium hybrids from Coulomb gauge QCD, Phys. Rev. D 78, 056003 (2008)

47. J. J. Dudek and E. Rrapaj, Charmonium in lattice QCD and the non-relativistic quark-model, Phys. Rev. D 78, 094504 (2008)

48. C. P. Shen, et al. (Belle Collaboration), Updated cross section measurement of $e^{+} e^{-} \rightarrow K^{+} K^{-} J / \psi$ and $K_{S}^{0} K_{S}^{0} J / \psi$ via initial state radiation at Belle, Phys. Rev. D 89(7), 072015 (2014)

49. T. Abe, et al. (Belle-II Collaboration), Belle II technical design report, arXiv: 1011.0352 [physics.ins-det]

50. Z. G. Zhao, Talk at the International Workshop on Physics at Future High Intensity Collider $2-7 \mathrm{GeV}$ in China, January 13-16, 2015, University of Science and Technology of China (USTC), Hefei, China 